\title{
INTRATUMORAL STAGES OF METASTATIC CELLS: A SYNTHESIS OF ONTOGENY, Rho/Rac GTPases, EPITHELIAL- MESENCHYMAL TRANSITIONS, AND MORE
}

\author{
by \\ Xosé R. Bustelo \\ Centro de Investigación del Cáncer and Instituto de Biología Molecular y Celular del \\ Cáncer, CSIC-University of Salamanca, Salamanca, Spain.
}

Running title: Early stages of metastasis

Key words: cancer, migration, cytoskeleton, stromal cells, inflammation

Total words in main text: 4,799

*To whom correspondence should be addressed:

Mailing address: Centro de Investigación del Cáncer, CSIC-University of Salamanca, Campus Unamuno s/n, 37007 Salamanca, Spain

E-mail: xbustelo@usal.es Phone: +34-923294802Ｆax: +34-923294743 


\section{ABSTRACT}

Metastasis is one of the clinical parameters that has a strong negative influence on the prognosis of cancer patients. In recent years, significant advances have furthered our understanding of this process at the molecular and biological levels. This review will discuss recent discoveries relating to the earliest, intra-tumoral stages of metastasis in cancer cells, specifically focusing on: (i) the development of metastatic traits during primary tumorigenesis; (ii) intrinsic and extrinsic cancer cell programs associated with malignant traits; (iii) the intra-tumoral migration patterns of cancer cells and the dynamic roles played by the Rho/Rac GTPases and epithelial-mesenchymal transitions in this process; and (iv) the genetic strategies used by metastatic cancer cells to promote intra-tumoral cell migration and their subsequent escape to peripheral tissues. Finally, the therapeutic and diagnostic relevance of this information will be discussed, as well as potential future developments.

\section{ABBREVIATIONS USED (in alphabetical order)}

Ahr, aryl hydrocarbon receptor; Ddr1, Discoidin domain receptor 1; Dicer, enzyme capable of "dicing" double stranded RNAs; Dock, dedicator of cytokinesis; EMT, epithelial-mesenchymal transition; EM/MET, epithelial-mesenchymal/mesenchymalepithelial transition; Eph, tyrosine kinase receptor expressed in an erythropoietinproducing hepatocellular cell line; Ephrin, Eph-receptor interacting protein; GAP, GTPase activating protein; GEF, guanosine nucleotide exchange factor; Hotair; homeobox transcript antisense RNA; Hoxd10, homeobox D10; IL, interleukin; MET, mesenchymalepithelial transition; MYBPH, myosin binding protein H; MMP, matrix metalloprotease; ncRNA, non-coding RNA; PTTG1, pituitary tumor-transforming 1; PyMT, polyomavirus middle T-antigen; RCP, Rab-coupling protein; Sec23a, Sec23 homolog A; TGF, transforming growth factor; VEGF, vascular endothelial growth factor; XIAP, X-linked inhibitor of apoptosis protein. 


\section{INTRODUCTION}

Metastasis, the biological process that encompasses the dissemination of cancer cells from the primary tumor and the subsequent formation of new tumor masses in peripheral tissues, is one of the main clinical parameters known to affect the prognosis of cancer patients nowadays. Indeed, its detection in patients is usually associated with resistance to chemotherapeutic treatments, higher post-treatment recurrence rates, and poor cancer patient survival rates $[1,2]$. Thus, the understanding of the metastatic process at both the genetic and biological level is of utmost importance to develop more effective anti-cancer therapies. Metastasis can be subdivided into a number of distinct stages according to mechanistic and regulatory criteria (Fig. 1). The first stage occurs within the primary tumor, where subpopulations of cancer cells develop a metastatic phenotype and migrate through a densely-packed environment composed of extracellular matrix, cancer cells and stromal cells in order to exit the tumoral mass (Fig. 1, point 1). The second stage involves the dissemination of cancer cells away from the primary tumor via either lymph vessels (Fig. 1, point 2) or the vasculature (a process called intravasation: Fig. 1, point 3). Circulating cancer cells (Fig. 1, point 4) then adhere to the microvasculature and move across the vascular endothelial cell layer and the extracellular matrix of the peripheral tissue in a process generically referred to as extravasation (Fig. 1, point 5). Finally, the extravasated cells colonize the new peripheral niche, a process known as macrometastasis (Fig. 1, point 6). Despite the malignancy associated with the metastatic process, it is in fact highly inefficient as it is estimated that less than $0.5 \%$ of cells that enter the circulation eventually form metastases. Indeed, most of the circulating cells die upon extravasation to non-permissive peripheral tissues (Fig. 1, point 8). Less frequently, cancer cells enter a 
dormant state in the peripheral niche either at the single-cell or micrometastasis stage (Fig. 1, point 7). When appropriate growth conditions arise, these cells exit the dormant state to form a macrometastasis (Fig. 1, point 9).

Recent reviews have focused on the history of the research carried out in this area, the conceptual frameworks that help understand the biological basis of this process, and the biological programs that regulate many of the stages of metastasis [1-5]. The present review will focus on issues specifically related to the earliest, intratumoral metastatic stages of cancer cells. Recent analyses of this particular phase of the metastatic process have shed light on the ontogeny of tumor metastasis, the necessary developmental and physiological conditions, and the biological programs that guide the overall metastatic process. These findings have also revealed new, and in some cases unexpected information about the migration of cancer cells within tumors, the regulation and role of Rho/Rac GTPases during cancer cell migration, and the dynamic roles of processes such as epithelial-mesenchymal transitions. Some of those findings also have significant potential to be translated into clinical applications, especially in the area of cancer diagnosis.

\section{The metastatic phenotype develops pre-selectively in the primary tumor mass}

Historically, two different models have been used to explain the ontogeny of metastasis. The "tumor-centric" model stated that all metastases originate from fully metastatic cells that are already present in the primary tumor. By contrast, the "multi-centric" theory postulated that cancer cells must undergo additional rounds of genetic alterations in peripheral niches to finally form the macrometastasis [5]. Although the latter model was at odds with standard Darwinian evolutionary theory, it conveniently explained a number of 
clinical observations, such as the long-term dormancy of metastatic cancer cells in healthy tissues and the frequent cases of patients displaying metastasis with no obvious physical indications of primary tumors. The recent sequencing of primary tumors and metastases from cohorts of patients unequivocally tilted the balance in favor of the "tumor-centric" model [6-10]. Irrespective of the tumor type analyzed, these studies consistently demonstrated that: (i) metastases originate monoclonally from single cancer cells originally present in the primary tumor; (ii) the metastatic populations are characterized by more extensive genetic/genomic alterations than other cancer cells present in the primary tumor; (iii) metastasis-specific mutations are also detected in discrete and regionalized cell subpopulations present in the primary tumors of the same patients, indicating that no metastatic niche-specific mutations occur; and (iv) there is a closer genetic proximity between metastases obtained from the same organ than in those retrieved from distinct anatomic locations, suggesting that metastasis requires tissue-specific biological programs (see below). Collectively, these findings indicate that metastatic traits are fully acquired at the primary tumor stage and that metastases originate late in tumor development as a consequence of increased genomic instability, thereby requiring a significant period to fully develop in patients (Fig. 2).

These observations have been applied to breast, pancreatic and prostate tumors, and will be probably extrapolated to other tumor types in coming years. If so, they will also influence the mechanistic interpretation of poorly characterized metastatic stages. For example, the acquisition of, and exit from, the dormant metastatic state will be better explained in terms of the absence/restoration of signaling cross-talk between the extravasated cancer cells and the microenvironment, rather than by the generation of new 
mutations in dormant cancer cells. Indeed, the data currently available indicate that a number of signaling routes involved in cell adhesion, mitogenesis and stress responses affect metastatic cancer cell dormancy (for review, see [11]). Notwithstanding the above evidence, the possibility that metastatic cancer cells from other tumor types could undergo a second wave of mutations during the dormant state cannot be formally disregarded as yet, especially in dormant states theoretically compatible with the generation and subsequent expansion of new pro-growth mutations (i.e., micrometastases that are kept dormant due to a steady-state equilibrium between rates of proliferation and apoptosis). The sequencing of additional tumor types should clarify this issue in the near future.

\section{Intrinsic cancer cell programs associated with the metastatic phenotype}

The genetic relatedness of metastases obtained from the same peripheral location suggests that genetically programmed routes pre-determine the colonization of specific peripheral tissues by metastatic cells (Fig. 2). This concept is in accordance with the "soil and seed" hypothesis originally proposed by Stephen Paget in 1889 to explain the tissue-specific dissemination of metastatic cancer cells [5]. This idea was recently confirmed following the identification of gene and ncRNA signatures directly associated with the tropism of metastatic cancer cells towards peripheral tissues, such as the brain, lung and bone [12-20]. The functional dissection of these signatures also led to the discovery of specific proteins and ncRNAs that play critical roles in step- and tissue-specific metastatic stages, including intravasation and extravasation, as well as the subsequent fitness of cancer cells in peripheral tissues [12-19,21-25] (Fig. 3).

Despite the large heterogeneity of biological programs that regulate the tissue- 
specific tropism of metastatic cancer cells, the pro-metastatic proteins and/or microRNAs can be subdivided in two main subclasses: the "metastastic maintenance" and the "metastatic virulence" groups. The former are regulatory factors (proteins and microRNAs) that play proactive roles in both primary tumorigenesis (i.e., proliferation, angiogenesis) and specific stages of the overall metastatic process (v.gr., vascular permeability, extravasation). The latter represent pro-metastatic factors that, surprisingly, have no significant role in primary tumorigenesis.

While the enrichment of genes that maintain the metastatic state in the primary tumor is conceptually logical in terms of the expansion of mutations conferring an advantageous phenotype within the overall cell population, the appearance of "virulent" pro-metastatic factors is more challenging. According to standard population genetics dynamics, cells expressing these factors would be progressively out-competed by cell clones containing genetic programs associated with better fitness within the primary tumor. Thus, how do cell clones harboring these gene signatures become enriched in the primary tumor? Although the answer to this question remains unclear, a recent study by Joan Massagué's group revealed that a significant percentage of the cells in the primary tumor may not actually originate in the tumor itself but rather, they are "prodigal son"-like cells that have returned to the primary tumor from the bloodstream or from peripheral metastatic nodules [26]. These cells were proposed to be the source of the virulence gene signatures present in the primary tumors, as they have undergone peripheral selection that favors the enrichment of cell clones carrying pro-metastatic mutations. Given the large estimated percentage of these "returning" cells in experimentally-induced tumors, this process was assumed to be genetically programmed. Indeed, the reseeding of the tumor by cancer cells 
is driven by the secretion of chemotactic factors (IL6, IL8) by the primary tumor, and it requires the upregulation of an extravasation-like program in the returning cancer cells through the secretion of MMPs and the expression of cytoskeletal components [26].

\section{Extrinsic cancer cell programs affect the latency and efficiency of metastasis}

Although the metastatic process was traditionally viewed from a purely cancer cell point of view, the acquisition of a metastatic phenotype is now understood as a dialectic process affected by the pre-tumoral developmental history of cancer cells and by ancillary influences emanating from diverse stromal cell types. These influences affect the speed at which metastatic traits can develop within the tumor, the effective migration/intravasation of the metastatic cells, and even the survival of the metastatic cells in distant peripheral tissues (Fig. 4). One of the best examples of the influence of cancer cell ontogeny on the development of the metastatic phenotype is the high and rapid rate of metastasis in melanoma, which can be explained by the strong expression of the EMT-promoting Slug transcriptional factor [27] in normal melanocytes [28]. The progression to the metastatic state in solid tumors also depends on the cooperative action of stromal cells, such as myoepithelial cells, adipocytes, immunosuppressive T-regulatory lymphocytes, myeloidderived suppressor cells, Gr1+ monocytes, M2 subtype macrophages and platelets [29,30] (Fig. 4). In the case of early stages of intratumoral metastasis, these non-tumor cells promote autocrine and paracrine loops that favor tumor progression [31,32], cancer cell motility [31-37], angiogenesis [38], and the evasion of anti-tumoral cytotoxic Tlymphocytes and natural killer cells [29,30,39]. Intratumoral stromal cells also favor extra- 
tumoral stages of metastasis by activating TGF $\beta$-dependent signaling, which in turn stimulates pro-permeability vascular programs that promote the intravasation and extravasation of cancer cells in specific peripheral tissues [17]. These cells also favor the secretion of extracellular matrix proteins by tumor cells that, upon release into circulation and attachment to peripheral tissues, create "pro-metastatic niches" in healthy tissues before the arrival of the metastatic cells [40] (Fig. 4).

Stromal cells are attracted to the tumor through passive signaling events that are triggered by pathological or extracellular matrix induced inflammation, and/or intratumoral hypoxia $[29,30,41,42]$. Alternatively, they can be actively enticed towards the tumor by the active secretion of chemokines and by the extracellular ligands expressed by cancer [4143], tumor-associated stromal and hematopoietic cells [35,37,44-46], and/or endothelial cells [38] (Fig. 4). Although these ancillary events would appear to be activated at relatively advanced stages of primary tumor development, this may not be the case for all tumor types. For example, sequencing of the genomes of hepatocellular carcinomas has shown that the "first wave" of mutations that originate in these tumors are related to the generation of a pro-inflammatory environment [47]. Moreover, in some cases the "prometastatic" inflammation may be generated by chance by a tumor non-autonomous program, due to changes in the normal environment in which the initial cancer cells are found (Fig. 4). This process has been recently demonstrated in the post-partum mammary gland, whereby the intrinsically high levels of extracellular matrix deposition, fibrosis and acute inflammation typically associated with that physiological state favor the acquisition of both migratory and invasive properties by breast cancer cells [48]. This physiological conditioning probably explains the poor prognosis associated with postpartum breast 
tumors detected in women [49].

\section{Cancer cells undergo dynamic changes in migration patterns during metastasis}

In addition to the acquisition of genetic traits that prepare metastatic cells for the biological and physical challenges they face outside the primary tumor, these cells must acquire the migratory and invasive capacity required for migration within the tumor, as well as for intravasation, extravasation and migration in the new peripheral niche. Although it was generally assumed that cancer cells migrated individually within tumors, recent evidence revealed that cancer cells adopt collective and single cell migratory behaviors inside tumors, in a dynamic and reversible manner. Furthermore, the signaling routes involved in these processes has altered previously held paradigms about the role played in this process of Rho/Rac GTPases, a Ras subfamily with key roles in the control of cytoskeletal dynamics $[50,51]$. Thus, in addition to the standard role of specific members of this subfamily in the generation of different cytoskeletal structures, such as filopodia (Cdc42), membrane ruffles (Rac1), lamellipodia (Rac1) and stress fibers (RhoA) [50,51], we now know that they also contribute to the acquisition of specific cell shapes and intercellular adhesion mechanisms. These processes are essential to explain how tumor cells migrate both within the tumor and when leaving the primary tumor mass.

The migratory behavior of cancer cells within the tumor is conditioned by the presence of extracellular signals in the surrounding environment. In inner regions of tumors that lack the factors that induce EMT, tumor cells tend to migrate in large multicellular clusters that follow the tracks in the extracellular matrix created by leading fibroblasts (Fig. 5, collective 1). This movement is coordinated by the activation of fibroblast and cancer 
cell specific signaling pathways. The movement of the leading fibroblast is driven by active RhoA/Rock-dependent actomyosin contractility, $\alpha_{3} / \alpha_{5}$-integrin signaling, force-mediated matrix remodeling and MMP activity (Fig. 5). By contrast, the trailing cancer cells depend on Cdc42-mediated actomyosin contractility [52] and on "gluing forces" that favor the cohesion of the entire migratory cell cluster (Fig. 5). This cohesion is generated by two independent mechanisms. On one hand, actomyosin contractility is inhibited in areas of cell-cell contact within the cell cluster, a process that involves the E-cadherin/Ddr1/RhoEdependent inactivation of Rho family GTPases [53] (Fig. 5). On the other hand, the cellcell contact zones are further glued together by the interaction of the EphA tyrosine kinase receptor and one of its ligands, eprhin-B2, both of which are present in adjacent cells of the cluster [54] (Fig. 5). This type of movement is essential for the dissemination of cancer cells to lymph nodes, although it is not required for the intravasation of cancer cells [55].

The migratory cancer cell cluster becomes scattered upon reaching peripheral tumor cell areas enriched with extracellular signals that favor EMT (Fig. 5). Depending on the signal transduction route and the Rho/Rac GTPase engaged at that moment, the scattered cancer cells can acquire an amoeboid- or mesenchymal-like migratory behavior (Fig. 5). The former involves high levels of RhoA/Rock-triggered actomyosin contractility that induces the moving cell to adopt a round, globular shape, while the latter is characterized by cells with a fibroblast-like morphology and is dependent on high levels of Rac1 activity, high MMP levels and weak actomyosin contractility [56] (Fig. 5). Cells can fluctuate between these amoeboid and mesenchymal migratory patterns by modulating their levels of RhoA, Rac1 and Cdc42 [57-59] (Fig. 5). Current data indicate that the movement of single cells within the tumor is critical for both the intravasation and extravasation stages $[55,60]$. 
Cancer cells have also been proposed to adopt other migratory behaviors. For example, in the early stages of PyMT-induced breast cancer, cancer cells exhibit a novel form of Rac1-dependent collective migration that, unlike that described above, does not rely on leading fibroblasts [61] (Fig. 5, collective 2). In these moving clusters, cancer cells lose their polarity but they conserve other epithelial features, such as the correct distribution of E-cadherin and $\beta$-catenin in the plasma membrane [61]. Interestingly, cells in the leading edge of the moving cluster do not appear to project filopodia or undergo membrane ruffling during the migration process [61]. This type of movement seems to be "inherited" from the migratory pattern seen in non-transformed mammary epithelial cells during pubertal mammary gland morphogenesis [61]. Additional data indicate that metastatic cells from different tumors may also display different migratory behaviors outside the primary tumor. Indeed, a recent study reported that melanoma and lung adenocarcinoma cells follow different migratory pathways once extravasated into the brain: the former move in close physical contact with the microvasculature, while the latter migrate at a distance from and totally independently of the brain blood vessels [62]. It is likely that the use of sophisticated intravital microscopy techniques in additional animal-based tumor models will reveal new migratory behaviors in cancer cells at both the primary tumor and peripheral sites.

\section{Cancer cells deregulate Rho/Rac-dependent routes through different mechanisms}

Given the important role of Rho/Rac proteins in tumorigenesis and cancer cell migration, it is unsurprising that cancer cells use multiple strategies to deregulate their activity. In addition to the standard activation by gene amplification or autocrine/paracrine loops, recent studies have shown that the levels of Rho/Rac GTPases, Rho/Rac activators and/or 
effectors, can be increased in cancer cells by impeding ubiquitination-dependent protein degradation [63-65], stimulating specific transcriptional factors (c-Myc, PTTG1, Ahr) [6668], epigenetically silencing negative regulatory molecules (e.g., $M Y B P H$, a gene encoding an inhibitory Rock binding protein) [69], inactivating tumor suppressor proteins (e.g., Hoxd10) via the overexpression of microRNAs (miR-10b) or long ncRNAs (Hotair) $[22,70]$, or by depleting anti-metastatic microRNAs (miR-31) $[23,24]$. Perhaps the most dramatic mechanism used by cancer cells to upregulate Rho/Rac-dependent pathways is through the direct alteration of microRNA synthesis following Dicer elimination in cancer cells. The loss of Dicer may be achieved by transcriptional repression (via the mutant p53dependent inactivation of Tap63, a p53 related protein that promotes Dicer gene transcription) [71] or by miR-103/107-mediated degradation of the Dicer transcript [72]. Whatever the means, this is probably quite a useful stratagem, as it is estimated that $\approx 20 \%$ of all the cellular microRNAs fulfill motility- or EMT-related functions [73]. The signaling output of Rho/Rac-dependent routes can also be fine tuned by other regulatory mechanisms, including: the sumoylation of Rac1, which favors the sustained activity of this GTPase [74]; c-Myc triggered transcriptional feedback loops that antagonize specific downstream effects of RhoA [75]; and cytoskeletal-dependent signaling loops that favor the generation of further waves of active Rac1 [76]. It is expected that additional regulatory mechanisms will be revealed as we extend our understanding of the gene signatures and biological programs associated with cell motility and invasion in different cell types.

\section{The cancer cell migratory machinery is connected to other tumorigenic routes}

In addition to altering the functional status of Rho/Rac-related pathways, cancer cells can 
manipulate the motility/adhesion machinery using signaling mechanisms that, unexpectedly, involve tumor suppressors, vesicle trafficking regulators or apoptosis-related proteins. For example, mutant p53 favors cell migration, loss of directionality and metastasis by promoting the active recycling of the EGF receptor and of $\alpha_{5} / \beta_{1}$ integrins at the leading edge of migrating cancer cells. This function of mutant p53 is mediated by the binding to, and subsequent inactivation of the p53-related TAp53 protein [77]. Although the mechanism is not fully understood, it has been shown that the inactivation of TAp63 promotes the interaction of $\alpha_{5} / \beta_{1}$ integrins with RCP, a Rab-coupling protein involved in membrane trafficking events. Consistent with this model, cancer cells lacking TAp63 show the same migratory phenotype than mutant p53-expressing cells. Conversely, the promigratory and pro-metastatic effects of mutant p53 can be eliminated in cancer cells by overexpressing TAp63 or depleting RCP [77]. The participation of the vesicle recycling machinery in the migration of cancer cells is further highlighted by the role of Rab25 and Rab5 in this process, two intracellular trafficking-related GTPases that promote the recycling of vesicular $\alpha_{5} / \beta_{1}$-integrin (Rab25) and Rac1 proteins (Rab5) back to the leading edge of cancer cells [78,79]. Accordingly, Rab family genes and RCP are frequently amplified in specific human tumors $[77,80]$. In the case of the apoptotic machinery, the survivin/XIAP complex can promote migration by inducing the NFKB-dependent expression of fibronectin and, as a consequence, sustained $\beta_{1}$-integrin, FAK and Src signaling in both cancer and stromal cells [81]. Given the functional connections between the aforementioned pathways, these data suggest that the deregulation of a single regulatory step has a significant impact on the overall activity of these cytoskeletal-related routes through the indirect stimulation of parallel pathways. Such a possibility could be further 
assessed in future studies using standard gain- and loss-of-function approaches.

\section{Fluctuations between epithelial and mesenchymal traits are important for efficient} metastasis

Characterization of the biological processes that favor cell motility has provided significant insight into the role and regulation of EMT in the context of tumors. As discussed above, it is now clear that this process is not constitutively activated in tumor cells but rather, it fluctuates dynamically during the migration of cancer cells depending on regional and extracellular cues that are heterogeneously distributed within the tumor. Likewise, recent data have shown that epithelial-mesenchymal/mesenchymal-epithelial transitions (EM/MET) also occur in circulating cancer cells and within the peripheral niche [82]. This ability to dynamically regulate EM/MET is also important for the fitness of metastatic cells, because it has been shown that cancer cells that cannot revert to an epithelial state upon the extravasation step cannot generate metastasis $[55,83,84]$. All those results suggest that the mesenchymal and epithelial state can contribute to metastasis in function of the location of the cancer cell. What are these pro-metastatic properties? In the case of EMT, recent reports indicate that its contribution to metastatic dissemination is more complex than simply promoting cell scattering via the downregulation of E-cadherin and cell-cell junction-related proteins [27]. Consistent with this view, it has been shown that the mesenchymal state is directly associated with the acquisition of undifferentiated, stem celllike phenotypes by cancer cells [85-89], the induction of angiogenesis [90] and the inhibition of anti-tumoral lymphocytes [42] (Fig. 6). The pro-angiogenic effects are mediated by the transcriptional activation of the Vegf gene by $\beta$-catenin, a transcriptional 
factor released from the plasma membrane upon the loss of E-cadherin [90]. The immunosuppressant effects are triggered by Snail1-dependent expression of thrombospondin1, an extracellular matrix protein that stimulates a subclass of immunosuppressor lymphocytes (regulatory $\mathrm{T}$ cells) and that inhibits antigen-presenting cells [42]. As indicated above, the acquisition of mesenchymal traits also favors intravasation over lymph node dissemination and the extravasation step $[55,60,82]$. Recent observations have also illustrated some of the pro-metastatic properties induced by MET. For example, miR-200c, a microRNA that maintains epithelial morphology by inhibiting the expression of Zeb family transcripts, is important to block the secretion of antimetastatic soluble factors (i.e., Igfbp4, TinaglI1) by the extravasated cancer cells. To carry out this Zeb- and E-cadherin-independent function, mir-200c targets the transcript for Sec23a, a protein involved in the anterograde transport of proteins from the endoplasmic reticulum to the Golgi apparatus [84]. Other members of the same microRNA family (e.g., miR-200a) are involved in promoting resistance to oxidative stress [91], a property that may be advantageous for cancer cells both in the primary tumor and in specific peripheral niches. Since the study of the pro-metastatic advantages conferred by the MET is still in its infancy, it is likely that additional functions will be revealed in the near future.

Multiple controls have developed during evolution to ensure that the EMT program is only activated under very specific physiological or developmental conditions. Thus, the activation of this program poses a significant challenge to cancer cells. In addition to the indirect activation of the program via EMT-promoting ligands secreted by cancer cells and stromal cells (e.g., TGF $\beta$ ), recent reports have shown that EMT can be activated by deregulating EMT signaling elements at the transcriptional [25,71], epigenetic/chromatin 
remodeling [70,92-94], non-coding (nc)RNA [25,72,95,96], microRNA biosynthesis (i.e., elimination of Dicer mRNA) [71,72] and protein translation [97] levels (Fig. 6). The mechanism(s) used by cancer cells to overcome this prima facie constitutive activation of the EMT program in order to restore the pro-metastatic epithelial state upon extravasation into peripheral niches remains unclear.

\section{Potential clinical value of early pro-metastatic factors and gene signatures}

What are the potential clinical applications of this new information? The answer to this question probably depends on what we want to achieve. The use of signaling elements involved in the early intratumoral stages of the metastatic cascade does not seem particularly valuable from a therapeutic point of view. This is because the most clinically relevant issue in patient treatment is how to tackle already established metastases rather than how to prevent them. However, some of the identified early pro-metastatic factors may be therapeutically useful if they play additional roles in maintaining macrometastasis in peripheral tissues. For example, it has been shown that the forced re-expression of miR-31, a microRNA whose downregulation is important in both early and late metastatic stages of cancer cells $[23,24]$ (Figs. 3), can induce the regression of lung macrometastasis in vivo [98]. This result underscores the importance of carrying out similar studies with other prometastatic factors using experimental conditions that mimic as close as possible clinically relevant conditions.

The most direct and rapid application of the information discussed here will probably be through the development of new diagnostic tools. Indeed, many metastasislinked gene and ncRNA signatures have been identified and have enough predictive power 
to stratify patients in terms of survival, disease recurrence and of the development of general or tissue-specific metastases. These signatures could be easily reformatted into either small/medium complexity microarrays, or quantitative reverse transcription-PCR kits, to analyze biopsies from cancer patients. Some of these signatures have in fact already reached a clinical setting in microarray format $[99,100]$. The application of these new genetic diagnostic kits will be important to ensure the selective administration of usually harsh anti-tumor therapies only to patients whose tumor biopsies reveal "metastaticfriendly" gene signatures.

\section{CONCLUSIONS AND OUTLOOK}

The data presented above highlight the significant advances that have been made in our understanding of the origin, regulation and mechanics of the earliest stages of the metastatic process in recent years. We have also unraveled many aspects of the biological programs associated with each metastatic stage, such as the manner in which cancer cells migrate within and beyond the original tumor mass, and the genetic alterations that contribute to form more robust metastases. Finally, we have learnt new information about the prometastatic functions of Rho/Rac GTPases, tumor suppressors, apoptotic regulators, and the EM/ME transitions. Most of those advances are potentially relevant for the development of new therapeutic and diagnostic tools in the near future. Despite this progress, much work lies ahead. For example, as most research to date has focused on very specific cancer subtypes (i.e., breast, lung), little is known about the pro-metastatic regulatory and effector routes in the majority of solid tumors. Similarly, because the migratory patterns of cancer cells have been mainly studied in cancer cell lines derived from specific tumor types, it 
remains unclear how primary metastatic cancer cells migrate in vivo. In the near future, the combination of signaling, high-throughput technologies and cell/animal models should help to identify new regulatory mechanisms and genetic alterations associated with this process. Finally, studies specifically designed to obtain clinical rather than pre-clinical information will be essential to push this field into the therapeutic arena. The conceptual, biological and technological developments achieved in recent years will undoubtedly facilitate such studies in the future.

\section{ACKNOWLEDGEMENTS}

Work in the author's lab is supported by the Spanish Ministry of Economy and Competiveness (SAF2009-07172 and RD06/0020/0001) and by grants from the Spanish Association Against Cancer. Spanish funding is co-sponsored by the European Union.

The author has no competing financial interests to declare. 


\section{REFERENCES}

1. Valastyan S, Weinberg RA. 2011. Tumor metastasis: molecular insights and evolving paradigms. Cell 147:275-92.

2. Gupta GP, Massague J. 2006. Cancer metastasis: building a framework. Cell 127:67995.

3. Chaffer CL, Weinberg RA. 2011. A perspective on cancer cell metastasis. Science (New York, N.Y 331:1559-64.

4. Nguyen DX, Bos PD, Massague J. 2009. Metastasis: from dissemination to organspecific colonization. Nat Rev Cancer 9:274-84.

5. Fidler IJ. 2003. The pathogenesis of cancer metastasis: the 'seed and soil' hypothesis revisited. Nat Rev Cancer 3:453-8.

6. Yachida S, Jones S, Bozic I, Antal T, et al. 2010. Distant metastasis occurs late during the genetic evolution of pancreatic cancer. Nature 467:1114-7.

7. Campbell PJ, Yachida S, Mudie LJ, Stephens PJ, et al. 2010. The patterns and dynamics of genomic instability in metastatic pancreatic cancer. Nature 467:1109-13.

8. Navin N, Kendall J, Troge J, Andrews P, et al. 2011. Tumour evolution inferred by single-cell sequencing. Nature 472:90-4.

9. Ding L, Ellis MJ, Li S, Larson DE, et al. 2010. Genome remodelling in a basal-like breast cancer metastasis and xenograft. Nature 464:999-1005.

10. Liu W, Laitinen S, Khan S, Vihinen M, et al. 2009. Copy number analysis indicates monoclonal origin of lethal metastatic prostate cancer. Nat Med 15:559-65.

11. Aguirre-Ghiso JA. 2007. Models, mechanisms and clinical evidence for cancer dormancy. Nat Rev Cancer 7:834-46. 
12. Kang Y, Siegel PM, Shu W, Drobnjak M, et al. 2003. A multigenic program mediating breast cancer metastasis to bone. Cancer Cell 3:537-49.

13. Kang Y, He W, Tulley S, Gupta GP, et al. 2005. Breast cancer bone metastasis mediated by the Smad tumor suppressor pathway. Proc Natl Acad Sci USA 102:1390914.

14. Minn AJ, Gupta GP, Siegel PM, Bos PD, et al. 2005. Genes that mediate breast cancer metastasis to lung. Nature 436:518-24.

15. Gupta GP, Nguyen DX, Chiang AC, Bos PD, et al. 2007. Mediators of vascular remodelling co-opted for sequential steps in lung metastasis. Nature 446:765-70.

16. Gupta GP, Perk J, Acharyya S, de Candia P, et al. 2007. ID genes mediate tumor reinitiation during breast cancer lung metastasis. Proc Natl Acad Sci USA 104:1950611.

17. Padua D, Zhang XH, Wang Q, Nadal C, et al. 2008. TGFbeta primes breast tumors for lung metastasis seeding through angiopoietin-like 4. Cell 133:66-77.

18. Tavazoie SF, Alarcon C, Oskarsson T, Padua D, et al. 2008. Endogenous human microRNAs that suppress breast cancer metastasis. Nature 451:147-52.

19. Bos PD, Zhang XH, Nadal C, Shu W, et al. 2009. Genes that mediate breast cancer metastasis to the brain. Nature 459:1005-9.

20. Oskarsson T, Acharyya S, Zhang XH, Vanharanta S, et al. 2011. Breast cancer cells produce tenascin $\mathrm{C}$ as a metastatic niche component to colonize the lungs. Nat Med 17:867-74.

21. Fang F, Turcan S, Rimner A, Kaufman A, et al. 2011. Breast cancer methylomes establish an epigenomic foundation for metastasis. Sci Transl Med 3:75ra25. 
22. Ma L, Teruya-Feldstein J, Weinberg RA. 2007. Tumour invasion and metastasis initiated by microRNA-10b in breast cancer. Nature 449:682-8.

23. Valastyan S, Benaich N, Chang A, Reinhardt F, et al. 2009. Concomitant suppression of three target genes can explain the impact of a microRNA on metastasis. Genes Devel 23:2592-7.

24. Valastyan S, Reinhardt F, Benaich N, Calogrias D, et al. 2009. A pleiotropically acting microRNA, miR-31, inhibits breast cancer metastasis. Cell 137:1032-46.

25. Ma L, Young J, Prabhala H, Pan E, et al. 2010. miR-9, a MYC/MYCN-activated microRNA, regulates E-cadherin and cancer metastasis. Nat Cell Biol 12:247-56.

26. Kim MY, Oskarsson T, Acharyya S, Nguyen DX, et al. 2009. Tumor self-seeding by circulating cancer cells. Cell 139:1315-26.

27. Acloque H, Adams MS, Fishwick K, Bronner-Fraser M, et al. 2009. Epithelialmesenchymal transitions: the importance of changing cell state in development and disease. J Clin Invest 119:1438-49.

28. Gupta PB, Kuperwasser C, Brunet JP, Ramaswamy S, et al. 2005. The melanocyte differentiation program predisposes to metastasis after neoplastic transformation. Nat Genet 37:1047-54.

29. Grivennikov SI, Greten FR, Karin M. 2010. Immunity, inflammation, and cancer. Cell 140:883-99.

30. Qian BZ, Pollard JW. 2010. Macrophage diversity enhances tumor progression and metastasis. Cell 141:39-51.

31. Fukuda A, Wang SC, Morris JPt, Folias AE, et al. 2011. Stat3 and MMP7 contribute to pancreatic ductal adenocarcinoma initiation and progression. Cancer Cell 19:441-55. 
32. Lesina M, Kurkowski MU, Ludes K, Rose-John S, et al. 2011. Stat3/Socs3 activation by IL-6 transsignaling promotes progression of pancreatic intraepithelial neoplasia and development of pancreatic cancer. Cancer Cell 19:456-69.

33. Wu Y, Deng J, Rychahou PG, Qiu S, et al. 2009. Stabilization of snail by NF-kappaB is required for inflammation-induced cell migration and invasion. Cancer Cell 15:41628.

34. Sanz-Moreno V, Gaggioli C, Yeo M, Albrengues J, et al. 2011. ROCK and JAK1 signaling cooperate to control actomyosin contractility in tumor cells and stroma. Cancer Cell 20:229-45.

35. Chen J, Yao Y, Gong C, Yu F, et al. 2011. CCL18 from tumor-associated macrophages promotes breast cancer metastasis via PITPNM3. Cancer Cell 19:541-55.

36. Toh B, Wang X, Keeble J, Sim WJ, et al. 2011. Mesenchymal transition and dissemination of cancer cells is driven by myeloid-derived suppressor cells infiltrating the primary tumor. PLoS Biol 9:e1001162.

37. Tan W, Zhang W, Strasner A, Grivennikov S, et al. 2011. Tumour-infiltrating regulatory $\mathrm{T}$ cells stimulate mammary cancer metastasis through RANKL-RANK signalling. Nature 470:548-53.

38. Mazzieri R, Pucci F, Moi D, Zonari E, et al. 2011. Targeting the ANG2/TIE2 axis inhibits tumor growth and metastasis by impairing angiogenesis and disabling rebounds of proangiogenic myeloid cells. Cancer Cell 19:512-26.

39. Gaziel-Sovran A, Segura MF, Di Micco R, Collins MK, et al. 2011. miR-30b/30d regulation of GalNAc transferases enhances invasion and immunosuppression during metastasis. Cancer Cell 20:104-18. 
40. Erler JT, Bennewith KL, Cox TR, Lang G, et al. 2009. Hypoxia-induced lysyl oxidase is a critical mediator of bone marrow cell recruitment to form the premetastatic niche. Cancer Cell 15:35-44.

41. Kim S, Takahashi H, Lin WW, Descargues P, et al. 2009. Carcinoma-produced factors activate myeloid cells through TLR2 to stimulate metastasis. Nature 457:102-6.

42. Kudo-Saito C, Shirako H, Takeuchi T, Kawakami Y. 2009. Cancer metastasis is accelerated through immunosuppression during Snail-induced EMT of cancer cells. Cancer Cell 15:195-206.

43. Yang L, Huang J, Ren X, Gorska AE, et al. 2008. Abrogation of TGF beta signaling in mammary carcinomas recruits Gr-1+CD11b+ myeloid cells that promote metastasis. Cancer Cell 13:23-35.

44. Schmid MC, Avraamides CJ, Dippold HC, Franco I, et al. 2011. Receptor tyrosine kinases and TLR/IL1Rs unexpectedly activate myeloid cell PI3kgamma, a single convergent point promoting tumor inflammation and progression. Cancer Cell 19:71527.

45. De Nardo D, De Nardo CM, Nguyen T, Hamilton JA, et al. 2009. Signaling crosstalk during sequential TLR4 and TLR9 activation amplifies the inflammatory response of mouse macrophages. J Immunol 183:8110-8.

46. Nieman KM, Kenny HA, Penicka CV, Ladanyi A, et al. 2011. Adipocytes promote ovarian cancer metastasis and provide energy for rapid tumor growth. Nat Med 17:1498-503. 
47. Tao Y, Ruan J, Yeh SH, Lu X, et al. 2011. Rapid growth of a hepatocellular carcinoma and the driving mutations revealed by cell-population genetic analysis of whole-genome data. Proc Natl Acad Sci USA 108:12042-7.

48. Lyons TR, O'Brien J, Borges VF, Conklin MW, et al. 2011. Postpartum mammary gland involution drives progression of ductal carcinoma in situ through collagen and COX-2. Nat Med 17:1109-15.

49. Stensheim H, Moller B, van Dijk T, Fossa SD. 2009. Cause-specific survival for women diagnosed with cancer during pregnancy or lactation: a registry-based cohort study. J Clin Oncol 27:45-51.

50. Bustelo XR, Sauzeau V, Berenjeno IM. 2007. GTP-binding proteins of the Rho/Rac family: regulation, effectors and functions in vivo. Bioessays 29:356-70.

51. Jaffe AB, Hall A. 2005. Rho GTPases: biochemistry and biology. Annu Rev Cell Dev Biol 21:247-69.

52. Gaggioli C, Hooper S, Hidalgo-Carcedo C, Grosse R, et al. 2007. Fibroblast-led collective invasion of carcinoma cells with differing roles for RhoGTPases in leading and following cells. Nat Cell Biol 9:1392-400.

53. Hidalgo-Carcedo C, Hooper S, Chaudhry SI, Williamson P, et al. 2010. Collective cell migration requires suppression of actomyosin at cell-cell contacts mediated by DDR1 and the cell polarity regulators Par3 and Par6. Nat Cell Biol 13:49-58.

54. Astin JW, Batson J, Kadir S, Charlet J, et al. 2010. Competition amongst Eph receptors regulates contact inhibition of locomotion and invasiveness in prostate cancer cells. Nat Cell Biol 12:1194-204. 
55. Giampieri S, Manning C, Hooper S, Jones L, et al. 2009. Localized and reversible TGFbeta signalling switches breast cancer cells from cohesive to single cell motility. Nat Cell Biol 11:1287-96.

56. Sahai E, Marshall CJ. 2003. Differing modes of tumour cell invasion have distinct requirements for Rho/ROCK signalling and extracellular proteolysis. Nat Cell Biol 5:711-9.

57. Sanz-Moreno V, Gadea G, Ahn J, Paterson H, et al. 2008. Rac activation and inactivation control plasticity of tumor cell movement. Cell 135:510-23.

58. Calvo F, Sanz-Moreno V, Agudo-Ibanez L, Wallberg F, et al. 2011. RasGRF suppresses Cdc42-mediated tumour cell movement, cytoskeletal dynamics and transformation. Nat Cell Biol 13:819-26.

59. Gadea G, Sanz-Moreno V, Self A, Godi A, et al. 2008. DOCK10-mediated Cdc42 activation is necessary for amoeboid invasion of melanoma cells. Curr Biol 18:1456-65.

60. Sahai E. 2007. Illuminating the metastatic process. Nat Rev Cancer 7:737-49.

61. Ewald AJ, Brenot A, Duong M, Chan BS, et al. 2008. Collective epithelial migration and cell rearrangements drive mammary branching morphogenesis. Dev Cell 14:57081.

62. Kienast Y, von Baumgarten L, Fuhrmann M, Klinkert WE, et al. 2010. Real-time imaging reveals the single steps of brain metastasis formation. Nat Med 16:116-22.

63. de la Vega M, Kelvin AA, Dunican DJ, McFarlane C, et al. 2011. The deubiquitinating enzyme USP17 is essential for GTPase subcellular localization and cell motility. Nat Commun 2:259. 
64. Wang HR, Ogunjimi AA, Zhang Y, Ozdamar B, et al. 2006. Degradation of RhoA by Smurf1 ubiquitin ligase. Methods Enzymol 406:437-47.

65. Chen Y, Yang Z, Meng M, Zhao Y, et al. 2009. Cullin mediates degradation of RhoA through evolutionarily conserved BTB adaptors to control actin cytoskeleton structure and cell movement. Mol Cell 35:841-55.

66. Chan CH, Lee SW, Li CF, Wang J, et al. 2010. Deciphering the transcriptional complex critical for RhoA gene expression and cancer metastasis. Nat Cell Biol 12:45767.

67. Liao YC, Ruan JW, Lua I, Li MH, et al. 2011. Overexpressed hPTTG1 promotes breast cancer cell invasion and metastasis by regulating GEF-H1/RhoA signalling. Oncogene, in press.

68. Carvajal-Gonzalez JM, Mulero-Navarro S, Roman AC, Sauzeau V, et al. 2009. The dioxin receptor regulates the constitutive expression of the vav3 proto-oncogene and modulates cell shape and adhesion. Mol Biol Cell 20:1715-27.

69. Hosono Y, Yamaguchi T, Mizutani E, Yanagisawa K, et al. 2011. MYBPH, a transcriptional target of TTF-1, inhibits ROCK1, and reduces cell motility and metastasis. EMBO J. 31:481-93.

70. Gupta RA, Shah N, Wang KC, Kim J, et al. 2010. Long non-coding RNA HOTAIR reprograms chromatin state to promote cancer metastasis. Nature 464:1071-6.

71. Su X, Chakravarti D, Cho MS, Liu L, et al. 2010. TAp63 suppresses metastasis through coordinate regulation of Dicer and miRNAs. Nature 467:986-90.

72. Martello G, Rosato A, Ferrari F, Manfrin A, et al. 2010. A MicroRNA targeting dicer for metastasis control. Cell 141:1195-207. 
73. Zhang H, Hao Y, Yang J, Zhou Y, et al. 2011. Genome-wide functional screening of miR-23b as a pleiotropic modulator suppressing cancer metastasis. Nat Commun 2:554.

74. Castillo-Lluva S, Tatham MH, Jones RC, Jaffray EG, et al. 2010. SUMOylation of the GTPase Rac1 is required for optimal cell migration. Nat Cell Biol 12:1078-85.

75. Sauzeau V, Berenjeno IM, Citterio C, Bustelo XR. 2010. A transcriptional cross-talk between RhoA and c-Myc inhibits the RhoA/Rock-dependent cytoskeleton. Oncogene 29:3781-92.

76. Castro-Castro A, Ojeda V, Barreira M, Sauzeau V, et al. 2011. Coronin 1A promotes a cytoskeletal-based feedback loop that facilitates Rac1 translocation and activation. EMBO J 30:3913-27.

77. Muller PA, Caswell PT, Doyle B, Iwanicki MP, et al. 2009. Mutant p53 drives invasion by promoting integrin recycling. Cell 139:1327-41.

78. Caswell PT, Spence HJ, Parsons M, White DP, et al. 2007. Rab25 associates with alpha5beta1 integrin to promote invasive migration in 3D microenvironments. Dev Cell 13:496-510.

79. Palamidessi A, Frittoli E, Garre M, Faretta M, et al. 2008. Endocytic trafficking of Rac is required for the spatial restriction of signaling in cell migration. Cell 134:135-47.

80. Mitra S, Cheng KW, Mills GB. 2011. Rab GTPases implicated in inherited and acquired disorders. Semin Cell Dev Biol 22:57-68.

81. Mehrotra S, Languino LR, Raskett CM, Mercurio AM, et al. 2010. IAP regulation of metastasis. Cancer Cell 17:53-64. 
82. Labelle M, Begum S, Hynes RO. 2011. Direct Signaling between Platelets and Cancer Cells Induces an Epithelial-Mesenchymal-Like Transition and Promotes Metastasis. Cancer Cell 20:576-90.

83. Dykxhoorn DM, Wu Y, Xie H, Yu F, et al. 2009. miR-200 enhances mouse breast cancer cell colonization to form distant metastases. PloS one 4:e7181.

84. Korpal M, Ell BJ, Buffa FM, Ibrahim T, et al. 2011. Direct targeting of Sec23a by miR-200s influences cancer cell secretome and promotes metastatic colonization. Nat Med 17:1101-8.

85. Shipitsin M, Campbell LL, Argani P, Weremowicz S, et al. 2007. Molecular definition of breast tumor heterogeneity. Cancer Cell 11:259-73.

86. Mani SA, Guo W, Liao MJ, Eaton EN, et al. 2008. The epithelial-mesenchymal transition generates cells with properties of stem cells. Cell 133:704-15.

87. Singh A, Settleman J. 2010. EMT, cancer stem cells and drug resistance: an emerging axis of evil in the war on cancer. Oncogene 29:4741-51.

88. Scheel C, Eaton EN, Li SH, Chaffer CL, et al. 2011. Paracrine and autocrine signals induce and maintain mesenchymal and stem cell states in the breast. Cell 145:926-40.

89. Wellner U, Schubert J, Burk UC, Schmalhofer O, et al. 2009. The EMT-activator ZEB1 promotes tumorigenicity by repressing stemness-inhibiting microRNAs. Nat Cell Biol 11:1487-95.

90. Ceteci F, Ceteci S, Karreman C, Kramer BW, et al. 2007. Disruption of tumor cell adhesion promotes angiogenic switch and progression to micrometastasis in RAFdriven murine lung cancer. Cancer Cell 12:145-59. 
91. Mateescu B, Batista L, Cardon M, Gruosso T, et al. 2011. miR-141 and miR-200a act on ovarian tumorigenesis by controlling oxidative stress response. Nat Med 17:1627-35.

92. Oh YM, Kwon YE, Kim JM, Bae SJ, et al. 2009. Chfr is linked to tumour metastasis through the downregulation of HDAC1. Nat Cell Biol 11:295-302.

93. Wang Y, Zhang H, Chen Y, Sun Y, et al. 2009. LSD1 is a subunit of the NuRD complex and targets the metastasis programs in breast cancer. Cell 138:660-72.

94. Han HJ, Russo J, Kohwi Y, Kohwi-Shigematsu T. 2008. SATB1 reprogrammes gene expression to promote breast tumour growth and metastasis. Nature 452:187-93.

95. Li QQ, Chen ZQ, Cao XX, Xu JD, et al. 2011. Involvement of NF-kappaB/miR-448 regulatory feedback loop in chemotherapy-induced epithelial-mesenchymal transition of breast cancer cells. Cell Death Differ 18:16-25.

96. Kress TR, Cannell IG, Brenkman AB, Samans B, et al. 2011. The MK5/PRAK kinase and Myc form a negative feedback loop that is disrupted during colorectal tumorigenesis. Mol Cell 41:445-57.

97. Hussey GS, Chaudhury A, Dawson AE, Lindner DJ, et al. 2011. Identification of an mRNP complex regulating tumorigenesis at the translational elongation step. Mol Cell 41:419-31.

98. Valastyan S, Chang A, Benaich N, Reinhardt F, et al. 2011. Activation of miR-31 function in already-established metastases elicits metastatic regression. Genes Devel 25:646-59.

99. van de Vijver MJ, He YD, van't Veer LJ, Dai H, et al. 2002. A gene-expression signature as a predictor of survival in breast cancer. New Eng J Med 347:1999-2009. 
100. Glas AM, Floore A, Delahaye LJ, Witteveen AT, et al. 2006. Converting a breast cancer microarray signature into a high-throughput diagnostic test. BMC Genomics 7:278. 


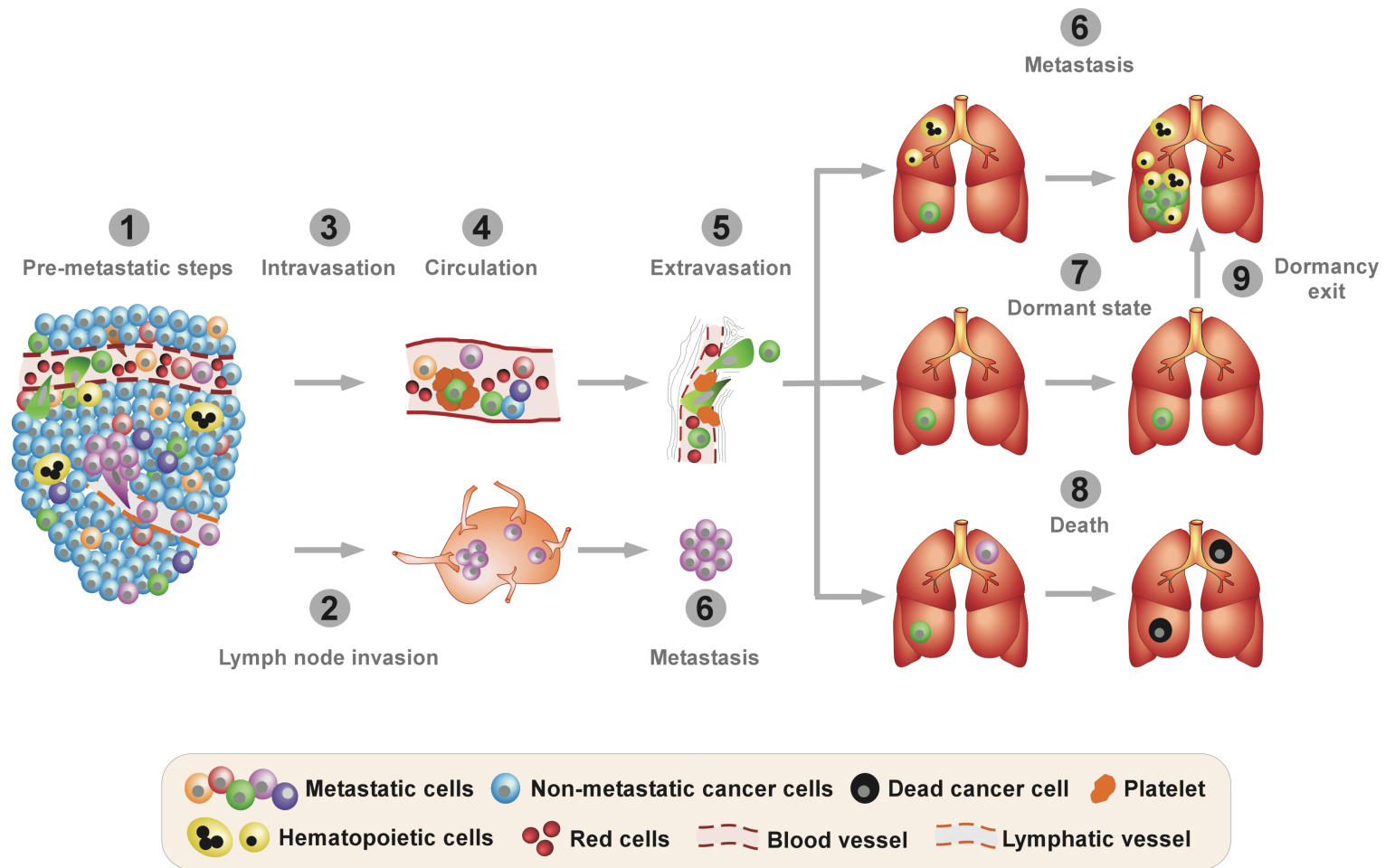

FIGURE 1. Summary of the stages followed by cancer cells to colonize peripheral tissues. Those include the pre-metastatic stages that take place in the primary tumor (step 1) and the subsequent stages of lymph node infiltration (step 2), intravasation (step 3), dissemination through the circulatory system (step 4), extravasation (step 5), macrometastasis formation (step 6), abortive metastasis (step 8), acquisition of a dormant state (step 7), and exit from dormancy (step 9). In the case of death in the peripheral tissue, we have included two different possibilities: (i) death of a cancer cell (shown in purple) with a proper extravasation program but lacking the right biological package to survive inside the lung parenchyma. (ii) death of a cancer cell (shown in green) that despite having the appropriate biological programs for extravasation and intraparenchymal growth, undergoes apoptosis due to deficient interactions with other cues present in the stroma of the peripheral tissue. 
For the sake of simplicity, we only illustrate the colonization of the lung by metastatic cells. The metastasis of cancer cells to other peripheral tissues may have different requirements. For example, the extravasation of cancer cells into the liver and bone does not appear to require specific biological programs due to the highly fenestrated nature of the vasculature in these tissues. Information about the cell types included in the figure is displayed in the inset at the bottom of the figure. Further details are provided in the main text. 
Bustelo (2012)

Figure 2

Bustelo

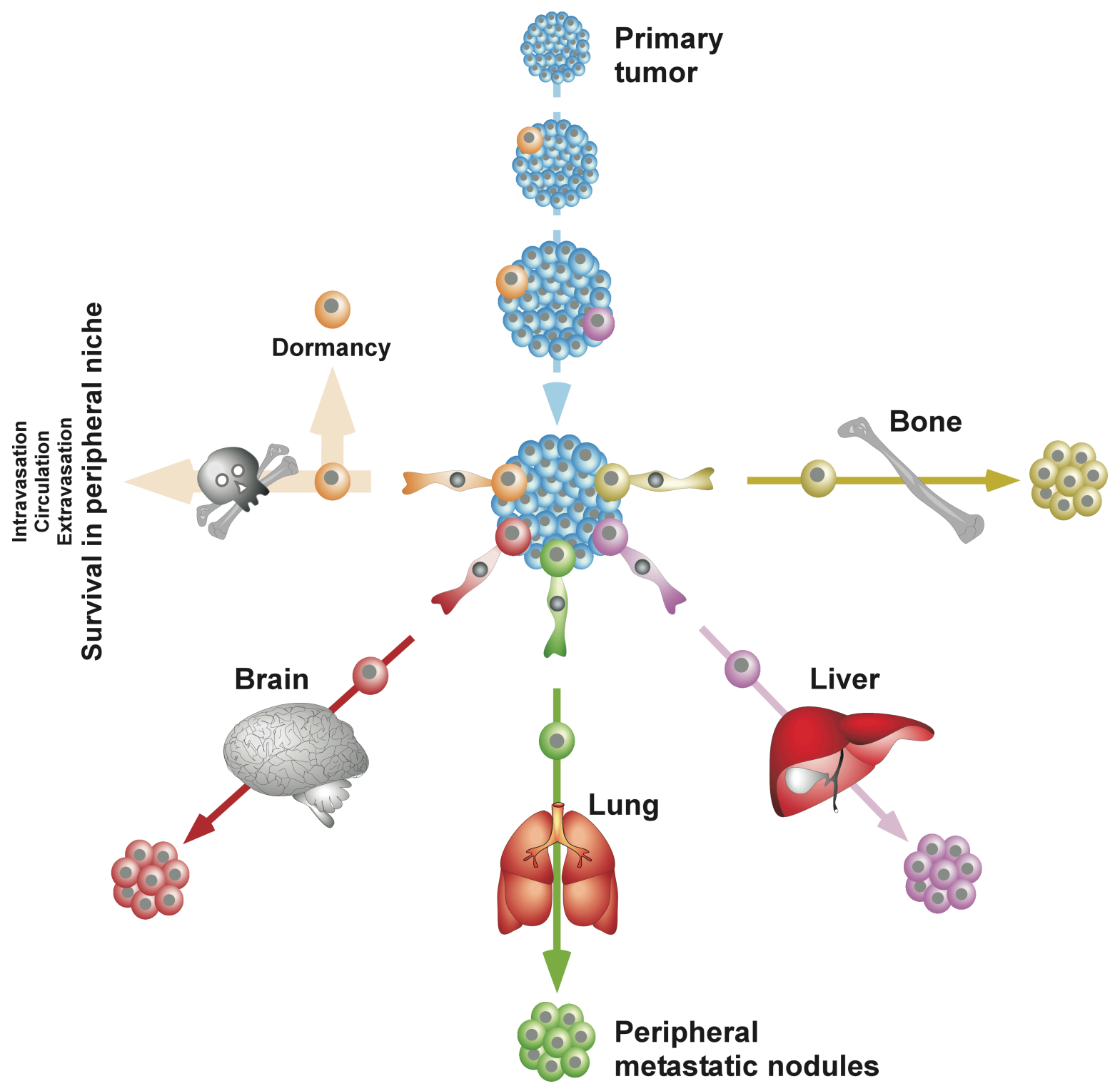

FIGURE 2. The evolution of metastatic traits in a primary tumor. According to recent data, the metastatic phenotype is acquired in late stages of cancer development due to increased genetic instability. Depending on the genetic and biological programs of the different metastatic clones, they will only disseminate to and successfully colonize a specific spectrum of peripheral tissues. However, most cells do not have the right biological program to survive through all stages, leading to the death of the vast majority of 
intravasated cancer cells. Current estimates indicate that the main selection step occurs upon the extravasation of cancer cells into the peripheral tissue. However, extravasated cells with the appropriate biological program to colonize peripheral tissues may enter a dormant state if they cannot find or induce permissive biological conditions for metastatic growth. 
Vascular permeability Intravasation

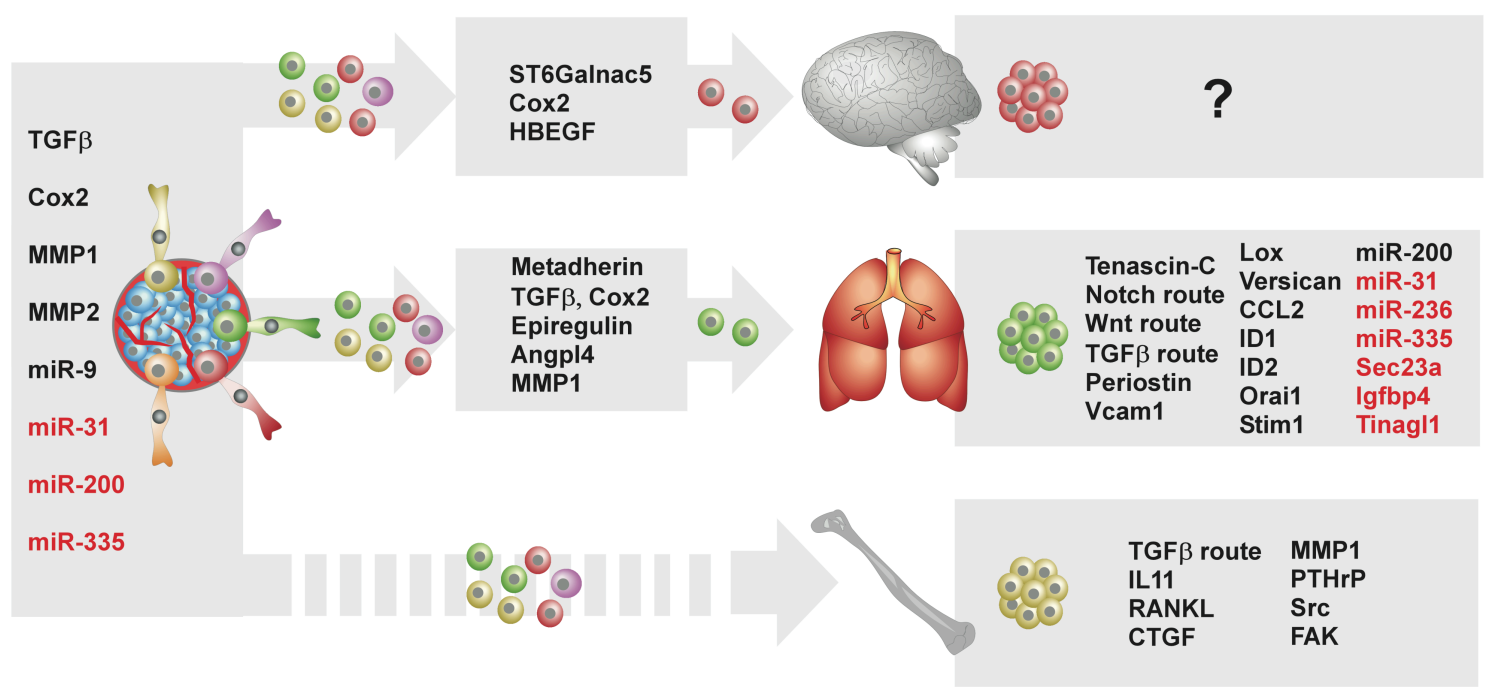

FIGURE 3. Some of the regulatory molecules known to affect the metastatic cycle of breast tumors. The main stages of metastasis are shown at the top. The peripheral tissues depicted include brain, lung and bone. Positive and negative regulators are shown in black and red, respectively. Please note that some molecules may have both positive and negative effects in different metastatic stages (e.g., the miR-200 family). A question mark indicates no available information, and lack of progress in this area has been mainly due to the technical challenges associated with analyzing metastatic stages inside the brain. The extravasation stage of metastatic cells to bone does not pose a significant biological challenge, as the bone vasculature is fenestrated and contains only endothelial cells with no associated mural cells. In normal conditions, such histological structure favors the continuous movement of hematopoietic cells in and out of the bone marrow. Angpl4, angiopoietin-related protein 4; CCL2, chemokine (C-C motif) ligand 2; Cox2, cyclooxygenase 2; CTGF, connective tissue growth factor; FAK, focal adhesion kinase; HBEGF, heparin-binding EGF-like growth factor; ID, inhibitor of DNA binding; Igfbp4, insulin-like 
growth factor binding protein 4; Lox, lysyl oxydase; Orai1, Orai calcium release-activated calcium modulator 1; PTHrP, parathyroid hormone related peptide; RANKL, receptor activator off nuclear factor kappa-B ligand; Stim1, stromal interactor molecule 1; ST6Galnac5, ST6 (alpha-N-acetyl-neuraminyl-2,3-beta-galactosyl-1,3)-N-acetylgalactosaminide alpha-2,6-sialyltransferase 5; Tinag11, tubulointerstitial nephritis antigenlike 1. Alternative names for those proteins can be found in the GeneCards database (www.genecards.org). Other abbreviations have been introduced in the main text. 
Figure 4

Bustelo

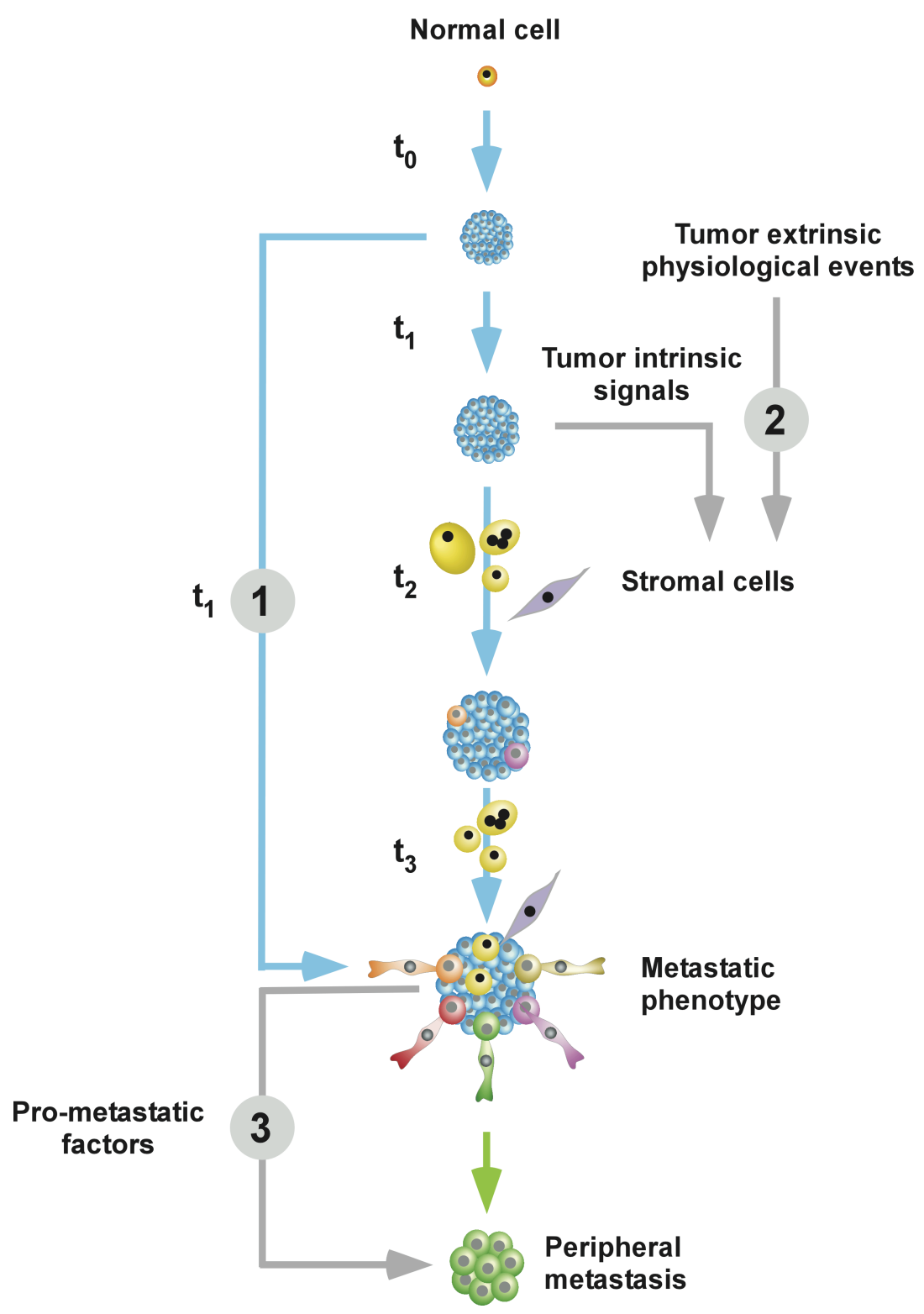

FIGURE 4. Factors affecting the development of metastatic traits in tumors. Nonmetastatic cancer cells are shown in blue. Metastatic cancer cells are shown in other colors to indicate their potential to colonize specific peripheral tissues. Stromal cells are shown in yellow. $t_{n}$, time involved for the development of step $n$ (depicted arbitrarily). As indicated in the main text, the rate at which metastasis develops can be altered by tumor-intrinsic and 
extrinsic factors. Extrinsic factors include the genetic background of the primary cell type in which the tumor originated (point 1), tumor-stimulated stromal cells, and physiological conditions of the individual during tumor development (e.g., inflammation triggered in the postpartum mammary gland; point 2). The secretion of factors by the primary tissue that, upon arrival and deposition in the peripheral niche, might favor the subsequent survival and growth of the extravasated cancer cells, is also depicted (point 3). 


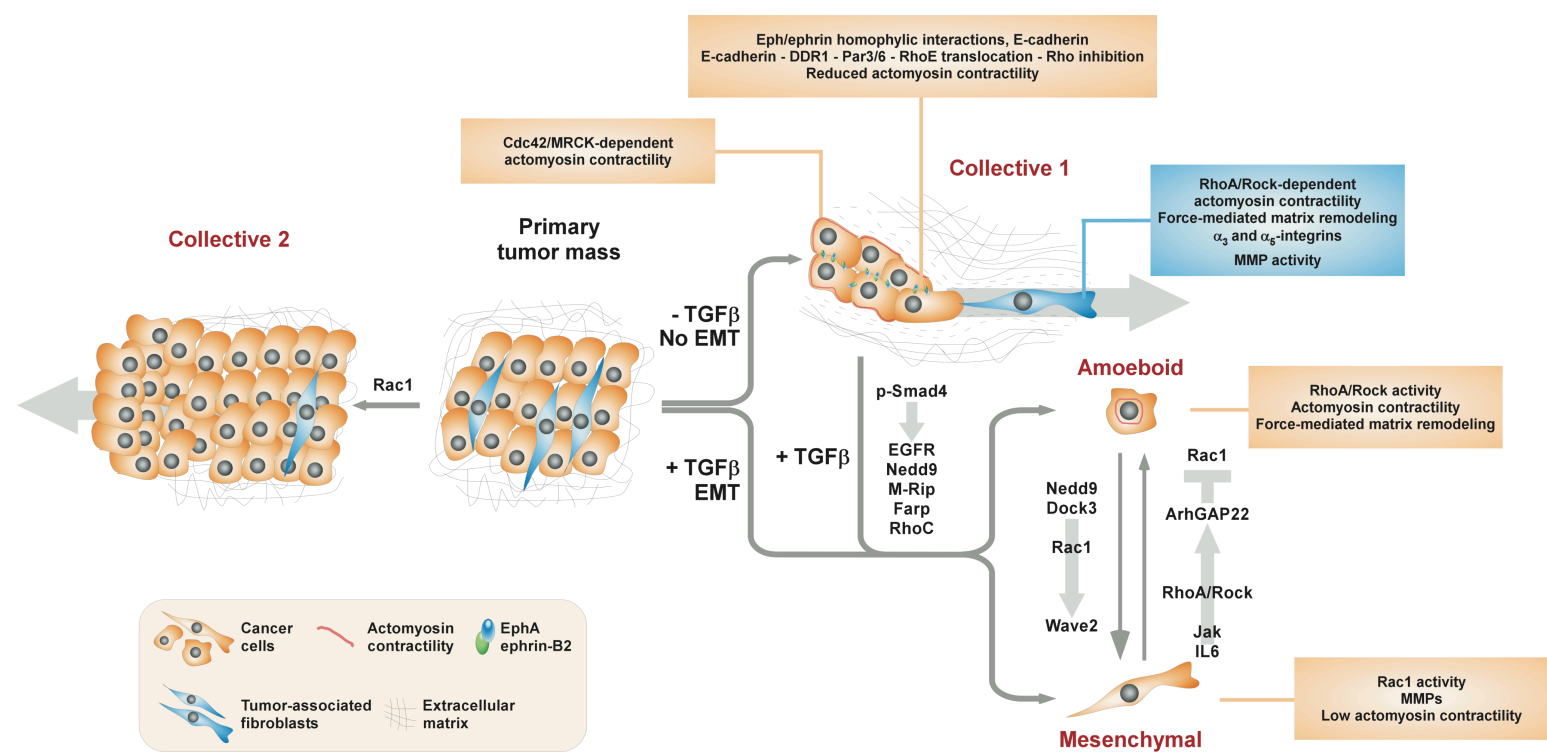

FIGURE 5. The four main types of movement described for cancer cells are highlighted in dark red (see main text): collective 1, collective 2, amoeboid and mesenchymal. Cancer cells and non-transformed, tumor associated-fibroblasts are shown in light brown and blue, respectively. The signaling routes involved in the migration of cancer cells and the leading fibroblast are indicated in the light brown and blue boxes, respectively. Transition of cancer cells from the collective 1 to the single cell migration type is mediated by TGF $\beta$-triggered EMT. This transition requires the activation of a complex phospho-Smad family protein 4 (p-Smad4)-dependent transcriptional program that includes the genes encoding the EGF tyrosine kinase receptor (EGFR), the adaptor molecule Nedd9 (neural precursor cell expressed, developmentally downs-regulated 9 protein, also known as: Casl -Crkassociated substrate-like-; Hef1; and $\left.\mathrm{p} 105^{\mathrm{Cas}}\right)$, the myosin phosphatase Rho-interacting protein (M-Rip), the Rac1 GEF Farp (FERM: four-point-one, ezrin, radixin, moesin; Rho GEF; and pleckstrin domain-containing protein), and the RhoC GTPase [52]. The transition from amoeboid to mesenchymal migratory behavior is dependent on a pathway involving 
Nedd9, the exchange factor Dock3 that catalyzes the activation (via GDP/GTP exchange) of the Rac1 GTPase, and the Rac1 downstream effector and cytoskeletal regulator Wave2 (Wiskott-Aldrich syndrome protein family member 2) [57]. Transition from the mesenchymal to the amoeboid type of movement requires the activation of RhoA, which in turn promotes the activation of the Rac1-specific ArhGAP2 in a Rock-dependent manner. The activation of ArchGAP22 by the RhoA/Rock axis leads to the inactivation of Rac1 by hydrolysis of its associated GTP into GDP [34,57]. Although the mechanism of RhoA activation remains to be elucidated, a recent study described its induction associated with inflammation via the IL6-dependent activation of Janus kinase (Jak) family members [34]. RasGRF2 (Ras GDP releasing factor 2), a Ras GEF that binds and blocks the activation of the Cdc42 GTPase, has also been implicated in the suppression of amoeboid movement [58]. Conversely, the Cdc42-specific Dock10 GEF promotes this type of movement. The latter two stages are not depicted in the figure. 


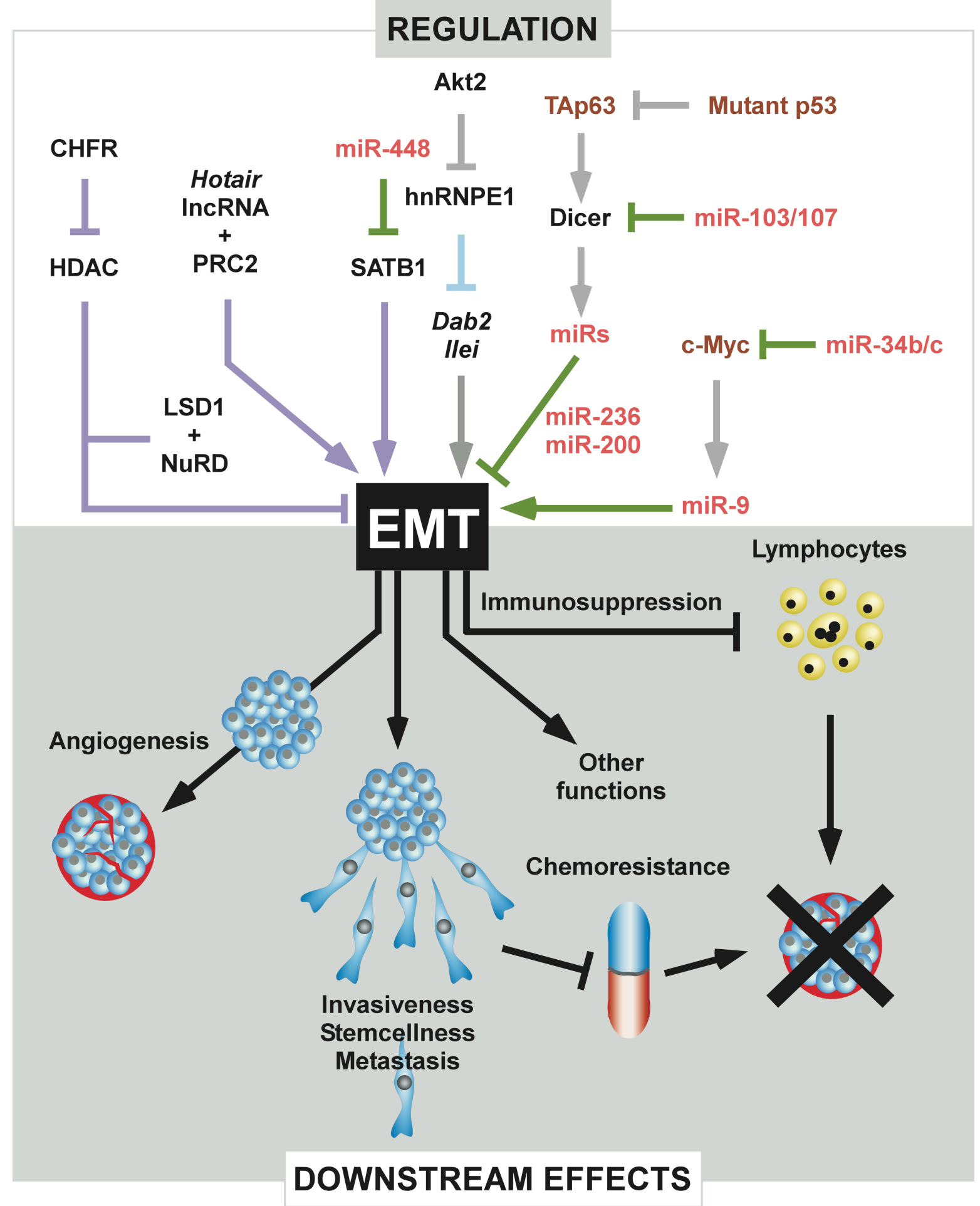

FIGURE 6. Examples of the new regulatory layers (top) and functions (bottom, shaded) associated with EMT regulatory pathways at the epigenetic (purple), translational (light 
blue) and microRNA (green) level. Transcription factors (brown) and microRNAs (light red) are also indicated. The microRNAs that activate the EMT usually target molecules implicated in the maintenance of the epithelial structure (e.g., E-cadherin), whereas microRNAs that work as inhibitors target pro-metastatic molecules (e.g., RhoC, RhoA, transcriptional factors involved in the EMT process). CHFR is a ubiquitin ligase involved in regulating mitotic checkpoints and in HDAC degradation. HDAC is a histone deacetylase 1 that epigenetically represses E-cadherin expression [92]. LSD1, lysinespecific demethylase 1 is involved in the NuRD complex [93], a Mi-2/nucleosome remodeling and deacetylase complex that controls the expression of elements in the TGF $\beta$ signaling pathway, and of other molecules involved in the EMT [93]. The Hotair ncRNA is involved in the assembly of the chromatin remodeling polycomb repressive complex 2 (PRC2) [70]. SATB1 (special AT-rich sequence-binding protein 1) is a chromosome remodeling factor whose overexpression in breast cancer leads to a large pro-EMT transcriptional program [94]. Dab2 (Disabled-2) and Ilei (interleukin-like EMT inducer) are two transcripts that lie downstream of EMT and whose translation is inhibited by the heterogeneous nuclear ribonucleoprotein E1 (hnRNPE1). This negative control is relieved by the Akt2-mediated phosphorylation of hnRNPE1 upon TGF $\beta$ stimulation [97]. Alternative designations for these proteins can be found in the GeneCards database (www.genecards.org). Activation and inactivation steps are shown as arrows and blunted lines, respectively. 